\title{
EXPANSION PROBLEMS OF ORDINARY LINEAR DIFFERENTIAL EQUATIONS WITH AUXILIARY CONDITIONS AT \\ MORE THAN TWO POINTS*
}

BY

\author{
CHARLES E. WILDER
}

\section{InTRODUCTION}

The boundary value and expansion problems for the equation of the $n$th order with boundary conditions at two points have been studied by Birkhoff. $\dagger$ Bôcher $\ddagger$ has suggested the generalization of these results to the equation with auxiliary conditions at more than two points. Such generalization of the essential properties of the differential system has been carried out by the author, and in this paper is given the proof of the convergence of the expansion, which may be studied quite independently of the other results. The formal development of the boundary problem and a more detailed discussion of the form of the series will be presented in other papers.

The differential equation is taken in the form

$$
\frac{d^{n} u}{d x^{n}}+*+p_{2} \frac{d^{n-2} u}{d x^{n-2}}+\cdots+p_{n} u+\lambda u=0
$$

in which $p_{2}, p_{3}, \cdots, p_{n}$, are functions of the real variable $x$, continuous together with their derivatives of all orders in the closed interval $(a, b)$. The auxiliary conditions apply to $k$ points of the interval $(a, b)$, of which the first and the last are the ends of the interval. These points, arranged in order of ascending algebraic magnitude, will be denoted by

$$
a_{1}=a, a_{2}, a_{3}, \cdots, a_{k-1}, a_{k}=b .
$$

* Presented to the Society, April 29, 1916.

$\dagger$ Birkhoff, Boundary value and expansion problems of ordinary linear differential equations, these Transactions, vol. 9 (1908), pp. 373-395. See also Tamarkine, Sur quelques points de la théorie des équations différentielles linéaires ordinaires et sur la généralisation de la série de Fourier, Rendiconti del Circolo Matematico di Palermo, vol. 34 (1912), pp. 345-382; and Birkhoff, Note on the expansion problems of ordinary linear differential equations, Rendicontidel Circolo Matematico di Palermo, vol. 36 (1913), pp. 115-126.

$\ddagger$ Bôcher, Boundary problems in one dimension, Proceedings of the Fifth International Congress of Mathematicians, Cambridge, 1913,vol. 1, pp. 163-195; see p. 166. 
The auxiliary conditions may then be written

$$
W_{i}(u) \equiv W_{1 i}(u)+W_{2 i}(u)+\cdots+W_{k i}(u)=0 \quad(i=1,2, \cdots, n),
$$

in which

$W_{j i}(u) \equiv \alpha_{j i} u\left(a_{j}\right)+\alpha_{j i}^{\prime} u^{\prime}\left(a_{j}\right)+\alpha_{j i}^{\prime \prime} u^{\prime \prime}\left(a_{j}\right)+\cdots+\alpha_{j i}^{(n-1)} u^{(n-1)}\left(a_{j}\right)$, and $\alpha_{j i}, \alpha_{j i}^{\prime}, \alpha_{j i}^{\prime \prime}, \cdots, \alpha_{j i}^{(n-1)}$, are constants, real or complex.

It is possible to define explicitly in terms of the auxiliary conditions and a fundamental system of solutions of the differential equation, a function $G(x, s ; \lambda)$, having the properties of a Green's function of the system. These properties will be discussed elsewhere with the exception of a single one, which forms the subject of this paper, namely that

$$
f(x)=\lim _{r=\infty} \frac{1}{2 \pi i} \int_{\Gamma} \int_{a}^{b} G(x, s ; \lambda) f(s) d s d \lambda,
$$

where $\Gamma$ is a contour in the $\lambda$-plane enclosing the first $r$ poles of $G(x, s ; \lambda)$, and $f(x)$ satisfies certain conditions of continuity, in some cases of the sort usually imposed, in others more restrictive, according to the nature of the auxiliary conditions. The auxiliary conditions themselves are subject to restrictions beyond those found necessary in the two point case. This integral may be regarded as the sum of the first terms of an infinite series of residues, corresponding to the series of orthogonal functions in the familiar cases, and these residues can in many cases be expressed linearly in terms of the characteristic functions of the differential system. Since the integral will be considered here on its own merits this interpretation will not be considered further.

\section{Preliminary formulas}

A fundamental system of solutions of the differential equation may be chosen analytic in $\lambda$. Let $y_{1}(x, \lambda), y_{2}(x, \lambda), \cdots, y_{n}(x, \lambda)$, be such a system. Then let $D(\lambda)$ be the determinant which has $W_{i}\left(y_{j}\right)$ for the element in the $i$ th row and $j$ th column. Its roots are the characteristic numbers for the system, but will concern us only as points where an expression having $D(\lambda)$ in its denominator ceases to be analytic. Let $D_{1}(s, \lambda)$ be the determinant which has the $(n-i)$ th derivative of $y_{j}(s)$ for the element in the $i$ th row and $j$ th column, and let $D_{2}(x, s ; \lambda)$ be this determinant with the elements in the first row replaced by the $y_{j}(x)$ with corresponding subscript. Then let

$$
\bar{G}(x, s ; \lambda)= \pm \frac{1}{2} D_{2} / D_{1}, \quad+\text { if } x>s, \quad-\text { if } x<s .
$$

If now $N(x, s ; \lambda)$ is the determinant formed by bounding $D(\lambda)$ on the top by the $y_{j}(x)$ with corresponding subscript, and on the right by the $W_{i}(\bar{G})$ 
with corresponding subscript, and filling in the upper right-hand corner with $\bar{G}(x, s ; \lambda)$, then the function $G(x, s ; \lambda)$ mentioned above is given by the formula

$$
G(x, s ; \lambda)=(-1)^{n} N / D .
$$

The proof of the convergence of the integral is based on the use of the following asymptotic solutions of the differential equation, due to Birkhoff.* Make the substitution $\lambda=\rho^{n}$. Then on any region $S$ of the $\rho$-plane, defined by the relation

$$
\frac{l \pi}{n} \leqq \arg \rho \leqq \frac{(l+1) \pi}{n},
$$

in which $l$ is a positive integer less than $n>1$, there exist $n$ linearly independent solutions, analytic in $\rho$, and such that

$$
\begin{aligned}
& y_{i}(x, \rho)=u_{i}(x, \rho)+e^{\rho x_{i}(x-a)} \frac{E_{1}}{\rho^{m}}, \\
& \frac{d y_{i}}{d x}=\frac{d u_{i}}{d x}+e^{\rho v_{i}(x-a)} \frac{E_{2}}{\rho^{m}-1}, \\
& \cdot \cdot \cdot \quad \cdot \quad \cdot \quad \cdot \quad \cdot \quad \cdot \quad \cdot \quad \cdot, \quad(i=1,2, \cdots, n), \\
& \frac{d^{n-1} y_{i}}{d x^{n-1}}=\frac{d^{n-1} u_{i}}{d x^{n-1}}+e^{\rho w_{i}(x-a)} \frac{E_{n}}{\rho^{m-n+1}},
\end{aligned}
$$

where

$$
u_{i}(x, \rho)=e^{\rho u_{i}(x-a)}\left\{1+\frac{u_{i 1}(x)}{\rho}+\cdots+\frac{u_{i m}(x)}{\rho^{m}}\right\} .
$$

Here $m$ is any positive integer, the $w$ 's are the roots of the equation

$$
w^{n}+1=0,
$$

the functions $u_{i j}(x)$ are finite and continuous for all values of $x$ in the interval $(a, b)$, and $E$ is a generic notation for functions of $\rho$ (and other variables) uniformly bounded for large values of $\rho$. In part of the work it will be necessary to use a more explicit form of the functions $u_{i j}(x)$, due to Milne, $\dagger$ viz., $u_{i j}(x)=\phi_{j}(x) /\left(w_{i}\right)^{j}$. The functions $\phi$ are then the same for all the $n$ solutions $y_{i}(x, \lambda)$.

By the substitution $\lambda=\rho^{n}$ the $\lambda$-plane goes over into two adjacent $S$ regions, $S_{1}$ and $S_{2}$. The integral (1) becomes

$$
\frac{1}{2 \pi i} \int_{\gamma_{1}} \int_{a}^{b} n \rho^{n-1} G\left(x, s ; \rho^{n}\right) f(s) d s d \rho
$$

$$
+{ }_{2 \pi i}^{1} \int_{\gamma_{2}} \int_{a}^{b} n \rho^{n-1} G\left(x, s ; \rho^{n}\right) f(s) d s d \rho,
$$

* Birkhoff, loc. cit., p. 381.

$\dagger$ Milne, Note on asymptotic expressions in the theory of linear differential equations, B u $11 \mathrm{e}-$ tin of the American Mathematical Society, vol. 23 (1917), pp. 166-169. 
in which $\gamma_{1}$ and $\gamma_{2}$ may be taken as arcs of the same circle with center at the origin.

Before substituting these solutions in the auxiliary conditions, the conditions are normalized as follows:

First, the number of conditions of order $n-1$ at the points $a$ and $b$ is reduced, by means of linear combinations, to two at most; then, the remaining conditions of order $n-2$ at these points are reduced to two at most; and so on.

It is convenient to introduce Birkhoff's notation, by which [ $w$ ] represents an expression of the form

$$
w+\frac{a}{\rho}+\frac{b}{\rho^{2}}+\frac{c}{\rho^{3}}+\cdots+\frac{E}{\rho^{m}}
$$

in which $w, a, b$, etc., are independent of $\rho$ but need not be constants, and $E$ is a function of $\rho$ and other variables bounded for large values of $\rho$.

The result of substituting the asymptotic solutions into the auxiliary conditions is

$$
\begin{aligned}
& W_{1 i}\left(y_{j}\right)=\left(\rho w_{j}\right)^{h_{1 i}}\left[\alpha_{1 i}\right] \text {, } \\
& W_{2 i}\left(y_{j}\right)=\left(\rho w_{j}\right)^{h_{2 i}}\left[\alpha_{2 i}\right] e^{\rho w_{j}\left(a_{2}-a\right)} \text {, } \\
& W_{k i}\left(y_{j}\right)=\left(\rho w_{j}\right)^{h_{k i}}\left[\alpha_{k i}\right] e^{\rho w_{j}(b-a)} .
\end{aligned}
$$

Because of the normalization of the auxiliary conditions $h_{1 i}=h_{k i} \leqq h_{j i}$, $j=2,3, \cdots, k-1$. So let us set $h_{1 i}=h_{k i}=h_{i}$, and we have $h_{i-1} \geqq h_{i}$ $\geqq h_{i+1}$, and no three successive $h_{i}$ 's are equal.

In the odd order case let $n=2 \mu-1$. Then the indices 1 to $n$ can be determined in such a way that for any $\rho$ in $S$

$$
R\left(\rho w_{1}\right) \leqq R\left(\rho w_{2}\right) \leqq R\left(\rho w_{3}\right) \leqq \cdots \leqq R\left(\rho w_{n}\right),
$$

where $R(w)$ indicates the real part of $w$. Hence for $S$

and it follows that

$$
\begin{array}{rr}
R\left(\rho v_{i}\right)<0 & (i=1,2, \cdots, \mu-1), \\
>0 & (i=\mu+1, \mu+2, \cdots, n), \\
=0 & \left(i=\mu \text { and } \arg \rho=\frac{l_{0}+\frac{1}{2}}{n} \pi\right),
\end{array}
$$

$$
\begin{aligned}
& W_{i}\left(y_{j}\right)=\left(\rho w_{j}\right)^{h_{i}}\left[\alpha_{1 i}\right] \\
& (j=1,2, \cdots, \mu-1), \\
& =\left(\rho u_{j}\right)^{h_{i}}\left[\alpha_{k i}\right] e^{\rho w_{j}(b-a)} \\
& (j=\mu+1, \mu+2, \cdots, n), \\
& =\left(\rho w_{j}\right)^{h_{i}}\left[\alpha_{1 i}\right]+\left(\rho w_{j}\right)^{h_{i}}\left[\alpha_{k i}\right] e^{\rho v_{j}(b-a)}+\sum_{l=2}^{k-1}\left(\rho w_{j}\right)^{h_{l i}}\left[\alpha_{l i}\right] e^{\rho w_{j}\left(a_{l}-a\right)} \\
& (j=\mu) .
\end{aligned}
$$


In the even order case let $n=2 \mu$. Then the indices 1 to $n$ can be determined in such a way that for any $\rho$ in $S$

$$
\begin{array}{rr}
R\left(\rho w_{i}\right)<0 & (i=1,2,3, \cdots, \mu-1), \\
>0 & (i=\mu+2, \cdots, n), \\
=0 & \left(i=\mu, \mu+1, \text { and either } \arg \rho=\frac{l_{0} \pi}{n} \text { or } \arg \rho=\frac{l_{0}+1}{n} \pi\right),
\end{array}
$$

and since $-w_{\mu}=w_{\mu+1}$, we have

$$
\begin{array}{rlr}
W_{i}\left(y_{j}\right)= & \left(\rho w_{j}\right)^{h_{i}}\left[\alpha_{1 i}\right] & (j=1,2, \cdots, \mu-1), \\
& =\left(\rho w_{j}\right)^{h_{i}}\left[\alpha_{k i}\right] e^{\rho w_{j}(b-a)} & (j=\mu+2, \cdots, n), \\
= & \left(\rho w_{\mu}\right)^{h_{i}}\left[\alpha_{1 i}\right]+\left(\rho w_{\mu}\right)^{h_{i}}\left[\alpha_{k i}\right] e^{\rho w_{\mu}(b-a)}+\sum_{l=2}^{k-1}\left(\rho w_{\mu}\right)^{h_{l i}}\left[\alpha_{l i}\right] \epsilon^{\rho \tau_{\mu}(a l-a)} \\
= & \left(-\rho w_{\mu}\right)^{h_{i}}\left[\alpha_{1 i}\right]+\left(-\rho w_{\mu}\right)^{h_{i}}\left[\alpha_{k i}\right] e^{-\rho w_{\mu}(b-a)} & (j=\mu), \\
& +\sum_{l=2}^{k-1}\left(-\rho w_{\mu}\right)^{h_{l i}}\left[\alpha_{l i}\right] e^{-\rho w_{\mu}(a l-a)} & (j=\mu+1) .
\end{array}
$$

\section{The function $D(\lambda)$}

Preparatory to taking up the main convergence problem it is necessary to study the distribution of those points where the function $D(\lambda)$ vanishes or becomes nearly equal to zero. This study is simplified by considering separately the cases where $n$ is odd and where $n$ is even.

Odd order case. When $n$ is odd, the substitution of the asymptotic expressions for the $W$ 's in the determinant $D(\lambda)$ gives

$$
D(\lambda)=\prod_{i=1}^{n} \rho^{h_{i}} \prod_{j=\mu+1}^{n} e^{\rho x_{j}(b-a)} \Delta,
$$

in which $\Delta$ is that determinant which has for the element in the $i$ th row and $j$ th column, $\left[\alpha_{1 i}\right] w_{j}^{h_{i}}$ for $j=1,2, \cdots, \mu-1$; $\left[\alpha_{k i}\right] w_{j}^{h_{i}}$ for $j=\mu+1, \mu+2$, $\cdots, n$, and finally

$$
B_{i} \equiv w_{\mu}^{h_{i}}\left(\left[\alpha_{1 i}\right]+\left[\alpha_{k i}\right] e^{\rho w_{\mu}(b-a)}+\sum_{l=2}^{k-1}\left(\rho w_{\mu}\right)^{h_{i i}-h_{i}}\left[\alpha_{l i}\right] e^{\rho w_{\mu}(a l-a)}\right),
$$

for $j=\mu$. Let $\nu$ be the highest power to which $\rho w_{\mu}$ enters in any $B_{i}$; then $\Delta$ can be expanded in the form

$$
\Delta \equiv\left[\theta_{1}\right]+\left[\theta_{k}\right] e^{\rho w_{\mu}(b-a)}+\left(\rho w_{\mu}\right)^{\nu}\left\{\sum_{l=2}^{k-1}\left[\theta_{l}\right] e^{\rho t v_{\mu}(a l-a)}\right\} .
$$

We shall now prove the

THEOREM. If in (6) neither $\theta_{1}$ nor $\theta_{k}$ is zero, and if for $\nu \geqq 1$ neither $\theta_{2}$ nor $\theta_{k-1}$ is zero, then $D(\lambda)$ has an infinite number of roots, and there exist two absolute constants $\omega$ and $\Omega$ such that the number of roots of $D(\lambda)$ in any circle 
about the origin of radius $r$ is less than $\Omega r$ and greater than $\omega r$ and, finally, for values of $\lambda$ uniformly away from the roots, $D(\lambda)$ remains uniformly away from zero.

This last statement is made more explicit by inequalities (16) and (21) below. We assume, then, that neither $\theta_{1}$ nor $\theta_{k}$ is zero. The number $\nu$ may be negative, zero, or positive. If it is negative $\Delta$ can be written more simply

$$
\left[\theta_{1}\right]+\left[\theta_{k}\right] e^{\rho w_{\mu}(b-a)} \text {. }
$$

Birkhoff* has shown that the roots of this are asymptotically equal to

$$
\rho_{l}=\frac{2 l \pi \sqrt{-1}}{w_{\mu}(b-a)}+\frac{\log \left(-\frac{\theta_{1}}{\theta_{k}}\right)}{w_{\mu}(b-a)},
$$

and that $\Delta$ remains uniformly away from zero if $\rho$ is uniformly away from a root.

When $\nu$ is zero, $\Delta$ becomes

$$
\left[\theta_{1}\right]+\left[\theta_{k}\right] e^{\rho w_{\mu}(b-a)}+\sum_{l=2}^{k-1}\left[\theta_{l}\right] e^{\rho w_{\mu}(a l-a)},
$$

and now at least one other $\theta$ in addition to $\theta_{1}$ and $\theta_{k}$ is assumed to be different from zero. In treating this case the distribution of roots of the function obtained by suppressing the brackets is determined, and then the roots of $\Delta$ are proved asymptotically equal to these.

It is convenient to make a change of variable. Let $\rho w_{\mu}\left(a_{2}-a\right)=z$, and divide through by $\theta_{1}$. The equation to be considered is, then,

$$
f(z) \equiv 1+Q_{0} e^{z}+Q_{1} e^{c_{1} z}+Q_{2} e^{c_{2} z}+\cdots+Q_{k-2} e^{c_{k-2} z}=0 .
$$

The $Q$ 's are complex constants, and in particular $Q_{k-2}$ and at least one other are different from zero. The $c$ 's are real constants such that $1<c_{1}<c_{2}$ $<\cdots<c_{k-2}$. Let $z=x+y i$, and let $R(\phi)$ denote the real part of $\phi$, and $I(\phi)$ the coefficient of the pure imaginary part. Let $\epsilon$ be an arbitrary small positive quantity, and choose $X$ so large that for $x=-X, f(z)$ differs from 1 by less than $\epsilon$, and at the same time for $x=X, f(z) / e^{c_{k-2} z}$ differs from $Q_{k-2}$ by less than $\epsilon$. The region between $x=X$ and $x=-X$ will be called the region $R$ throughout this paper. We shall suppose that $\epsilon$ is chosen small enough so that $f(z)$ does not vanish outside of $R$.

Now $f(z)$ is analytic in any finite region. Hence in any interval, no matter how small, along the $y$-axis, it is possible to find a line $y=$ constant along which $f(z)$ does not vanish. Let $y=Y_{1}$ and $y=Y_{2}$ be any two such lines. The number of roots of $f(z)$ in the rectangle $x=X, y=Y_{1}, x=-X$, $y=Y_{2}$ is determined by following the change in $\operatorname{arc} f(z)$ as $z$ describes this

\footnotetext{
* Birkhoff, loc. cit., pp. 383-5.
} 
rectangle. Now

and for $y$ constant $I(f)$ has the form

$$
\operatorname{arc} f(z)=\sin ^{-1} \frac{I(f)}{|f|},
$$

$$
I(f) \equiv d_{0} e^{x}+d_{1} e^{c_{1} x}+d_{2} e^{c_{2} x}+\cdots+d_{k-2} e^{c_{k-2} x},
$$

in which the $d$ 's are real constants. The finite roots of $I(f)$ are the same as those of

$$
I_{0}(f) \equiv d_{0}+d_{1} e^{\left(c_{1}-1\right) x}+d_{2} e^{\left(c_{2}-1\right) x}+\cdots+d_{k-2} e^{\left(c_{k-2}-1\right) x} .
$$

But between any two roots of this is a root of the derivative

$I_{0}^{\prime}(f) \equiv d_{1}\left(c_{1}-1\right) e^{\left(c_{1}-1\right) x}+d_{2}\left(c_{2}-1\right) e^{\left(c_{2}-1\right) x}$

while its roots are the same as those of

$$
+\cdots+d_{k-2}\left(c_{k-2}-1\right) e^{\left(c_{k-2}-1\right) x}
$$

$$
I_{1}(f) \equiv d_{1}\left(c_{1}-1\right)+d_{2}\left(c_{2}-1\right) e^{\left(c_{2}-c_{1}\right) x}+\cdots+d_{k-2}\left(c_{k-2}-1\right) e^{\left(c_{k-2}-c_{1}\right) x} .
$$

The argument is repeated till finally $I_{k-2}$ is reached, which has only one term and does not vanish for $x$ finite. Thus $I(f)$ has at most $(k-2)$ finite roots. Should any of the I's have multiple roots the argument must be slightly changed, but the result is, of course, the same.

Now as $z$ traces the line $x=-X, f(z)$ remains very nearly equal to 1 , so that the resulting change in $\operatorname{arc} f(z)$ is less than some small positive quantity $\eta_{1}$ which approaches zero with $\epsilon$. As $z$ traces the line $x=X$ the change in $\operatorname{arc} f(z)$ differs but slightly from $c_{k-2}\left(Y_{2}-Y_{1}\right)$, as is at once apparent from the formula

$$
\operatorname{arc} f(z)=\operatorname{arc} e^{c_{k-2^{z}}}+\operatorname{arc} \frac{f(z)}{e^{c_{k-2} z}} .
$$

In other words, the change in arc along this line can be written

$$
c_{k-2}\left(Y_{2}-Y_{1}\right) \pm \eta_{2},
$$

where $\eta_{2}$ is a small positive quantity which approaches zero with $\epsilon$. Finally along the lines $y=Y_{1}$ and $y=Y_{2}$ the function $\sin \{\operatorname{arc} f(z)\}$ vanishes at most $k-2$ times, whence $\operatorname{arc} f(z)$ increases by less than $(k-1) \pi+\eta_{3}$ and $(k-1) \pi+\eta_{4}$ respectively, where $\eta_{3}$ and $\eta_{4}$ are again small positive quantities which approach zero with $\epsilon$. To sum up, as $z$ traces the rectangle the resulting change in $\operatorname{arc} f(z)$ is not greater than

nor less than

$$
c_{k-2}\left(Y_{2}-Y_{1}\right)+2(k-1) \pi+\eta
$$

$$
c_{k-2}\left(Y_{2}-Y_{1}\right)-2(k-1) \pi-\eta \quad\left(\eta=\eta_{1}+\eta_{2}+\eta_{3}+\eta_{4}\right),
$$

if $Y_{2}-Y_{1}$ is large enough to make this expression positive. Since $\eta$ can be 
made arbitrarily small by taking $\epsilon$ small enough, it can be dropped from these inequalities. So finally in any interval of $R$ of length $l$ the number of roots of $f(z)$ is not greater than $c_{k-2} l / 2 \pi+k-1$, nor less than $c_{k-2} l / 2 \pi-k+1$, if this number is positive. In particular, by making $l$ very small, one sees that a multiple root of $f(z)$ cannot be of order higher than $k-1$.

It is now necessary to prove that if $z$ remains uniformly away from a root of $f(z)$ then $f(z)$ remains uniformly away from zero. This is proved by means of the following auxiliary theorem:

Theorem. Given $f\left(z, x_{1}, x_{2}, \cdots, x_{n}\right)$, continuous in all of its arguments and analytic in $z$ for $a_{i} \leqq x_{i} \leqq b_{i}$ and $z$ in a closed finite region $S$ of the complex plane, and such that for no set of values of the $x$ 's is the number of roots of $f(z)$ greater than a given constant $N$; if for any set of values of the $x$ 's, $z$ is a point at a distance greater than $\delta$ from a zero of $f(z)$ and from the boundary of $S$, then

$$
\left|f\left(z, x_{1}, x_{2}, \cdots, x_{n}\right)\right| \geqq \lambda,
$$

where $\lambda$ is a real positive constant independent of the $x$ 's.

It is no restriction to assume that $\delta$ is small enough so that for.each set of values of the $x$ 's the corresponding region of variation for $z$, defined by the hypotheses, actually contains points. This follows from the assumption that for any set of values of the $x$ 's the number of roots of $f(z)$ is less than $N$. Now for any particular set of values of the $x$ 's there is a $\lambda$, such that for these $x$ 's and for $z$ restricted to its corresponding region of variation,

$$
\left|f\left(z, x_{1}, x_{2}, \cdots, x_{n}\right)\right| \geqq \lambda,
$$

while there is a value of $z$ for which the equality holds. We have thus defined a function of the $x$ 's, $\lambda\left(x_{1}, x_{2}, \cdots, x_{n}\right)$, which, by means of the easily proved fact that the zeros of $f\left(z, x_{1}, x_{2}, \cdots, x_{n}\right)$ are continuous functions of the $x$ 's, can be shown to be continuous in the closed region $a_{i} \leqq x_{i} \leqq b_{i}$. Since $\lambda\left(x_{1}, x_{2}, \cdots, x_{n}\right)$ is continuous in a closed region, it has a lower limit which it attains. Call this limit $\lambda$. Then for some value of $z$ and the $x$ 's, $|f|=\lambda$. Hence $\lambda$ is not equal to zero, for $f$ does not vanish in any of the regions for which the function of which $\lambda$ is the lower limit was defined. Hence the theorem is proved.

First apply this theorem to the function

$$
f\left(z, \phi_{1}, \phi_{2}, \cdots, \phi_{k-2}\right) \equiv 1+Q_{0} e^{z}+Q_{1} e^{c_{1} z+\phi_{1} i}+\cdots+Q_{k-2} e^{c_{k-2} z+\phi_{k-2} i} .
$$

The region $S$ can be that portion of $R$ for which $0 \leqq y \leqq 2 \pi$, augmented by a strip of width $\delta$ surrounding it. Obviously the breadth of $R$, that is, the value of $X$, depends only on the $Q$ 's and not on the $\phi$ 's. It is also clear that the function satisfies the conditions of the theorem. Let $\lambda$ indicate the number determined by the theorem corresponding to $\delta$. 
Consider, then, the function (8) in $R$. Any interval of $R$,

$$
2 l \pi \leqq y \leqq 2(l+1) \pi,
$$

can, by a transformation $\bar{y}=y+2 l \pi$ be brought to the interval $0 \leqq y \leqq 2 \pi$, and $f$ becomes

$$
f(z+2 l \pi i) \equiv 1+Q_{0} e^{z}+Q_{1} e^{c_{1} z-2 l \pi c_{1} i}+\cdots+Q_{k-2} e^{c_{k-2} z-2 l \pi c_{k-2} i} .
$$

But, without changing $f$, each expression $2 l \pi c_{j} i$ can be replaced by a $\phi_{j} i$ where $0 \leqq \phi \leqq 2 \pi$. So $f(z+2 l \pi i)$ has the form above, and for $z$ at a greater distance from a root than $\delta,|f(z+2 l \pi)| \geqq \lambda$. By giving $l$ all integral values, all of $R$ is covered by these intervals, and so finally, for $z$ in $R$ and at a distance from a root of $f(z)$ greater than $\delta,|f(z)| \geqq \lambda>0$.

We can now prove that the roots of (7) are asymptotically equal to those of (8). First it is clear that by choosing $\epsilon$ small enough we can be sure that all the roots of (7) lie within the region $R$. Let us denote (7) by $\psi(\rho)$ and (8) by $f(\rho)$. Then in $R$

$$
\psi(\rho) \equiv \theta_{1} f(\rho)+\frac{E}{\rho},
$$

from which it is obvious that the roots of $\psi(\rho)$ are very nearly equal to those of $f(\rho)$ for large values of $\rho$. Now

whence

$$
\psi(\rho) \equiv \theta_{1} f(\rho)\left(1+\frac{E}{\theta_{1} f(\rho) \rho}\right),
$$

$$
\operatorname{arc} \psi(\rho) \equiv \operatorname{arc} \theta_{1}+\operatorname{arc} f(\rho)+\operatorname{arc}\left(1+\frac{E}{\theta_{1} f(\rho) \rho}\right)
$$

Let $\delta$ be an arbitrary small positive quantity. Then according to the previously determined distribution of the roots of $f(\rho)$, it is possible to enclose these roots in groups of less than $k$ in simply connected regions of major diameter less than $k \delta$, and such that no point of the boundary is at a distance from a root of $f(\rho)$ less than $\delta$. On the boundaries of these regions we have then $|f(\rho)| \geqq \lambda$, where $\lambda$ is determined by the theorem above. Hence for large values of $\rho$, the expression $E /\left(\theta_{1} f(\rho) \rho\right)$ is very small on these boundaries, and so the resulting change in arc $\left(1+E /\left(\theta_{1} f(\rho) \rho\right)\right)$ as $z$ traces the boundary of a region is zero, and the change in $\operatorname{arc} \psi(\rho)$ is the same as the change in $\operatorname{arc} f(\rho)$. So the number of roots of $\psi(\rho)$ in any such region is for large values of $\rho$ the same as the number of roots of $f(\rho)$ in it; that is, the roots of $\psi(\rho)$ are asymptotically equal to those of $f(\rho)$.

From this together with (9) can be deduced the fact that for large values of $\rho$ in $R$ the function $\psi(\rho)$ remains uniformly away from zero when $\rho$ is uniformly away from a root of $\psi(\rho)$. Outside of $R$ the function $\psi(\rho)$ of course remains uniformly away from zero. 
It is convenient to consider next the case in which $\Delta$ reduces to

$$
\left[\theta_{1}\right]+\left[\theta_{k}\right] e^{\rho w_{\mu}(b-a)}+\left(\rho w_{\mu}\right)^{\nu}\left[\theta_{2}\right] e^{\rho w_{\mu}\left(a_{2}-a\right)},
$$

which, in particular, it always does if $k=3$. It is assumed that none of the $\theta$ 's are zero, and that $\nu \geqq 1$. Then

where

$$
\Delta \equiv\left[\theta_{2}\right] e^{\rho w_{\mu}\left(a_{2}-a\right)} \Delta_{0},
$$

$$
\Delta_{0} \equiv\left(\rho w_{\mu}\right)^{\nu}+\left[\theta_{b}\right] e^{\rho w_{\mu}\left(b-a_{2}\right)}+\left[\theta_{a}\right] e^{-\rho w_{\mu}\left(a_{2}-a\right)},
$$

and in this

$$
\left[\theta_{a}\right] \equiv \frac{\left[\theta_{1}\right]}{\left[\theta_{2}\right]}, \quad\left[\theta_{b}\right] \equiv \frac{\left[\theta_{k}\right]}{\left[\theta_{2}\right]} .
$$

It is necessary to consider separately the part of $S$ in which $R\left(\rho w_{\mu}\right)$ is positive and the part in which it is negative.

$R\left(\rho w_{\mu}\right) \leqq 0$. It will now be shown that in that part of $S$ where $R\left(\rho w_{\mu}\right)$ $\leqq 0$, the roots of $\Delta_{0}$ are asymptotically equal to those of

$$
f(\rho) \equiv\left(\rho w_{\mu}\right)^{\nu}+\theta_{a} e^{-\rho w_{\mu}\left(a_{2}-a\right)} .
$$

To find the roots of $f(\rho)$ set $\rho w_{\mu}=x+y i=r e^{i \phi}$, and $-\theta_{a}=r_{a} e^{i \phi_{a}}$. When $f(\rho)$ is equated to zero the second term may be transposed, and then, when absolute values and angles are equated, the two following equations result:

$r^{\nu}=r_{a} e^{x\left(a_{2}-a\right)}, \quad \nu \phi=\phi_{a}-y\left(a_{2}-a\right)+2 m \pi \quad(m=0, \pm 1, \pm 2, \pm 3, \cdots)$.

The zeros of $f(\rho)$ are the points of intersection of these two curves. Since, in that part of the $\rho$ plane under consideration, $x \leqq 0, y>0$, the first curve is equivalent to

$$
y=\sqrt{r_{a}^{2 / \nu} e^{-2 x\left(a_{2}-a\right) / \nu}-x^{2}},
$$

and this has but one branch which is in character much like the exponential curve, and which for large values of $\rho$ approaches parallelism to the line $R\left(\rho w_{\mu}\right)=0$, i. e.,

It is also easily shown that

$$
\lim _{\rho=\infty}(d x / d y)=0 .
$$

$$
\lim _{\rho=\infty}(x / y)^{2}=0,
$$

which is to say that for points on this curve

$$
\lim _{\rho=\infty} \phi=\pi / 2+2 l \pi \quad(l=0, \pm 1, \pm 2, \pm 3, \cdots) .
$$

When this is substituted in the second curve it is found that the roots of $f(\rho)$ are asymptotically equal to points whose $y$ coördinates are given by

$$
y=\frac{\phi_{a}+2 m \pi-\nu\left(\frac{1}{2} \pi+2 l \pi\right)}{a_{2}-a},
$$


and since $\nu$ is an integer this is equivalent to

$$
y=\frac{\phi_{a}+2 m \pi-\frac{1}{2} \nu \pi}{a_{2}-a} .
$$

So the roots of $f(\rho)$ lie along the curve

$$
\left|\left(\rho w_{\mu}\right)^{v}\right|=\left|\theta_{a} e^{-\rho w_{\mu}\left(a_{2}-a\right)}\right|
$$

at intervals whose lengths approach $2 \pi /\left(a_{2}-a\right)$ as $\rho$ becomes infinite. For large values of $\rho$ these roots are all simple, for it is obvious that $f(\rho)$ and $f^{\prime}(\rho)$ cannot both vanish for the same value of $\rho$ when $\rho$ is large.

In order to prove that in that part of $S$ where $R\left(\rho w_{\mu}\right) \leqq 0$ the roots of $\Delta_{0}$ are asymptotically equal to those of $f(\rho)$, it is necessary to show that $|f(\rho)|$ remains uniformly away from zero when $\rho$ is uniformly away from a root. For this purpose let $T$ be the region included between the curves defined by the relation

$$
\left|\left(\rho w_{\mu}\right)^{\nu}\right|=\left|\theta_{a} e^{-\rho t o_{\mu}\left(a_{2}-a\right) \pm \sigma}\right| .
$$

Let $U$ be the region between $T$ and the line $R\left(\rho w_{\mu}\right)=0$, and let $V$ be the remainder of $S$ for which $R\left(\rho w_{\mu}\right)<0$. Choose $\sigma$ as any convenient positive real number. The following inequalities are immediate:

$$
\begin{array}{ll}
\text { for } U, & \left|\left(\rho w_{\mu}\right)^{\nu}\right| \geqq e^{\sigma}\left|\theta_{a} e^{-\rho w_{\mu}\left(a_{2}-a\right)}\right|, \\
\text { for } V, & \left|\left(\rho w_{\mu}\right)^{\nu}\right| \leqq e^{-\sigma}\left|\theta_{a} e^{-\rho w_{\mu}\left(a_{2}-a\right)}\right| ;
\end{array}
$$

from which it follows that

$$
\begin{array}{ll}
\text { for } U, & |f(\rho)| \geqq\left(e^{\sigma}-1\right)\left|\theta_{a} e^{-\rho w_{\mu}\left(a_{2}-a\right)}\right|, \quad \text { or } \quad\left(1-e^{-\sigma}\right)\left|\left(\rho w_{\mu}\right)^{\nu}\right|, \\
\text { for } V, & |f(\rho)| \geqq\left(1-e^{-\sigma}\right)\left|\theta_{a} e^{-\rho w_{\mu}\left(a_{2}-a\right)}\right| .
\end{array}
$$

For $\rho$ in $T$ it is necessary to proceed differently. Let $\rho_{0}$ be any root of $f(\rho)=0$, and call those points of $T$ which are at least as near to $\rho_{0}$ as to any other root of $f(\rho)=0$, the domain of $\rho_{0}$. Now the strip $T$ is asymptotically of constant width, and it is easily shown that this width is $2 \sigma /\left(a_{2}-a\right)$. On the other hand the distance between two roots of $f(\rho)=0$ was shown to be asymptotically equal to $2 \pi /\left(a_{2}-a\right)$, so that finally if $\rho_{0}$ is far enough out the distance of any point of the domain of $\rho_{0}$ from $\rho_{0}$ will be less than $2(\sigma+\pi) /\left(a_{2}-a\right)$. In this domain $f(\rho)$ can be written

$$
\begin{aligned}
f(\rho) \equiv f(\rho)-f\left(\rho_{0}\right) \equiv\left(\rho w_{\mu}\right)^{\nu}-\left(\rho_{0} w_{\mu}\right)^{\nu}+\theta_{a} e^{-\rho \omega_{\mu}\left(a_{2}-a\right)}-\theta_{a} e^{-\rho_{0} \omega_{\mu}\left(a_{2}-a\right)} \\
\equiv w_{\mu}^{\nu}\left(\rho-\rho_{0}\right)\left(\rho^{\nu-1}+\rho^{\nu-2} \rho_{0}+\cdots+\rho_{0}^{\nu-1}\right) \\
\quad+\theta_{a} e^{-\rho w_{\mu}\left(a_{2}-a\right)}\left(1-e^{\left(\rho-\rho_{0}\right) w_{\mu}\left(a_{2}-a\right)}\right) .
\end{aligned}
$$

In $T$ the absolute value of the first term is less than

$$
\frac{2(\sigma+\pi)}{a_{2}-a} \nu\left|\rho_{0}+\frac{2(\sigma+\pi)}{a_{2}-a}\right|^{\nu-1},
$$


and for sufficiently large values of $\rho$ this is less than a constant times $\left|\rho_{0}\right|^{\nu-1}$. Moreover, since in $T,\left|\rho w_{\mu}\right| v$ is between two constant multiples of $\left|e^{-\rho w_{\mu}\left(a_{2}-a\right)}\right|$, it follows that the first term is less than a constant times

$$
\left|e^{-[(\nu-1) / \nu] \rho w_{\mu}\left(a_{2}-a\right)}\right| \text {. }
$$

In the parenthesis in the last term let

$$
\left(\rho-\rho_{0}\right) w_{\mu}=r(\cos \phi+i \sin \phi), \quad \text { where } \quad-\pi \leqq \phi \leqq \pi .
$$

The parenthesis becomes

$$
\left(1-e^{r\left(a_{2}-a\right)(\cos \phi+i \sin \phi)}\right),
$$

which can vanish only at the points given by

$$
\cos \phi=0, \quad \sin \phi=\frac{2 l \pi}{r\left(a_{2}-a\right)}
$$

and the point $\rho_{0}$. In the domain of $\rho_{0}$ there is only one of these points, namely $\rho_{0}$ itself, and for that portion of the domain whose points are at a distance not less than $\delta>0$ from $\rho_{0}$ the absolute value of the parenthesis as a function of $r$ and $\phi$ is continuous and positive in a closed region. Hence it has a positive minimum which may be denoted by $\eta$. The last term of (13) is then greater than a constant times

$$
\left|e^{-\rho w_{\mu}\left(a_{2}-a\right)}\right|
$$

for that portion of the domain of $\rho_{0}$ at a distance from $\rho_{0}$ greater than $\delta$. This, combined with the inequality on the first term, gives the result that for large values of $\rho$ in $T$ and at a distance from a root of $f(\rho)=0$ greater than or equal to $\delta>0$,

$$
|f(\rho)|>\left|e^{-\rho \omega_{\mu}\left(a_{2}-a\right)}\right| K_{0},
$$

where $K_{0}$ is a positive constant. Similar inequalities have been proved for any $\rho$ in $U$ or $V$, so that this, with a new constant $K_{1}$, may be assumed to hold for all that part of $S$ for which $R\left(\rho v_{\mu}\right) \leqq 0$ for large values of $\rho$ at a distance from a root greater than or equal to $\delta$.

From (10) is obtained the relation

$$
\Delta_{0}=f(\rho)+\frac{E}{\rho} e^{-\rho x_{\mu}\left(a_{2}-a\right)}+\left[\theta_{b}\right] e^{\rho \mu_{\mu} \mu^{\left(b-a_{2}\right)}},
$$

which can be factored into

$$
\Delta_{0}=f(\rho)\left(1+\frac{E}{\rho} \frac{e^{-\rho x_{\mu}\left(a_{2}-a\right)}}{f(\rho)}+\frac{\left[\theta_{b}\right] e^{\rho x_{\mu}\left(b-a_{2}\right)}}{f(\rho)}\right) .
$$

Consider the portions of $T, U$, and $V$, bounded by two circular arcs $|\rho|=\rho^{\prime}$ and $|\rho|=\rho^{\prime \prime},\left(\rho^{\prime}<\rho^{\prime \prime}\right)$, such that no point of either arc is at a distance less than $\delta$ from a root of $f(\rho)=0$. Call such a region $\bar{S}$. It will be shown, 
by considering the change in arc $\Delta_{0}$ as $\rho$ traces the boundary of $\bar{S}$, that the number of roots of $\Delta_{0}$ and of $f(\rho)$ in $\bar{S}$ is the same, provided the innermost boundary of $\bar{S}$ is taken far enough out. Consider first the last term of the parenthesis in (15). Throughout the region $\bar{S}$ the numerator is bounded. In $U$ the denominator, $f(\rho)$, can be replaced by $\left(1-e^{-\sigma}\right)\left(\rho w_{\mu}\right)^{\nu}$ from inequality (12), and so for large values of $\rho$ in $U$ this last term is small. From the second of the inequalities (12) it can similarly be shown that this last term is also small for large values of $\rho$ in $V$, while from inequality (14) the same result is obtained for the boundary lines of $\bar{S}$ where they cross $T$, provided the innermost boundary of $\bar{S}$ is far enough out. On the other hand, from inequalities (12) and (14) the expression $e^{-\rho w_{\mu}\left(a_{2}-a\right)} / f(\rho)$ is bounded on the boundary of $\bar{S}$, and when it is multiplied by $E / \rho$ the result is small when the innermost boundary of $\bar{S}$ is far enough out. Under these conditions, then, the parenthesis in (15) can be written as $1+\eta$, where $\eta$ is small enough so that the arc of $1+\eta$ will return to its original value when $\rho$ traces the boundary of $\bar{S}$. It follows that the change in arc $\Delta_{0}$ is the same as the change in $\operatorname{arc} f(\rho)$, so that $\Delta_{0}$ and $f(\rho)$ have the same number of roots in $\bar{S}$.

Now exactly the same result is obtained if $\rho$ traces the circumferences of circles of arbitrarily small radius about the roots of $f(\rho)$, and this, combined with the previous result, shows that in that part of the region $S$ for which $R\left(\rho w_{\mu}\right) \leqq 0$, the roots of $\Delta_{0}$ are asymptotically equal to those of $f(\rho)$.

An important inequality for $\Delta$ can be deduced from formula (15). For $\rho$ large and at a distance from a root greater than $\delta$ inequality (14) holds. For $T$ and $V$ the exponential can be replaced by a constant times $\left(\rho w_{\mu}\right)^{\nu}$, while for $U$ the resulting inequality for $f(\rho)$ has already been obtained in (12). The parenthesis in (15) can be made nearly equal to 1 , and in particular, greater than $1 / 2$, by taking $\rho$ large enough; so that the inequality is obtained:

$$
\left|\Delta_{0}\right|>\left|\rho w_{\mu}\right|^{\nu} K_{2},
$$

in which $K_{2}$ is a constant depending only on $\delta$. The result of applying this to $\Delta$ is (see $(10))$

$$
|\Delta|>\left|\left(\rho u_{\mu}\right)^{\nu} e^{\rho w_{\mu}\left(a_{2}-a\right)}\right| K_{3},
$$

in which $K_{3}$ is a constant depending only on $\delta$. This holds for $\rho$ in that part of $S$ where $R\left(\rho w_{\mu}\right)$ is less than or equal to zero and greater than some fixed $\bar{\rho}$, and at a distance from a root of $f(\rho)$ at least as great as $\delta$. Equally important is the result, easily deducible from the same formulas, that under the same conditions $\Delta$ is greater than a constant depending only on $\delta$.

$R\left(\rho w_{\mu}\right) \geqq 0$. By entirely similar reasoning it is found that for that part of $S$ where $R\left(\rho w_{\mu}\right) \geqq 0$ the roots of $\Delta$ are asymptotically equal to those of

$$
\left(\rho u_{\mu}\right)^{\nu}+\theta_{b} e^{\rho r r_{\mu}\left(b-a_{*}\right)},
$$


which lie along the curve

$$
\left|\left(\rho w_{\mu}\right)^{\nu}\right|=\left|\theta_{b} e^{\rho t 0_{\mu}\left(b-a_{2}\right)}\right|
$$

at intervals whose lengths approach $2 \pi /\left(b-a_{2}\right)$, and which are all simple for large values of $\rho$. Inequalities on $f(\rho), \Delta_{0}$, and $\Delta$, similar to those above are found also in this case.

Finally the odd order case with $k$ points is in general, i. e., when certain constants do not vanish, merely a combination of the cases already considered. The characteristic equation $\Delta=0$ is as given in (6). The coefficient of $\left(\rho w_{\mu}\right)^{\nu}$ can be brought to the form (8) by dividing by $e^{\rho w_{\mu}\left(a_{2}-a\right)}$ and setting $\rho w_{\mu}\left(a_{3}-a_{2}\right)=z$. It is now assumed that none of the constants $\theta_{1}, \theta_{2}, \theta_{k-1}, \theta_{k}$, is zero. It follows then, that the roots of this coefficient are all in a certain region $R$ lying along the line $R\left(\rho w_{\mu}\right)=0$, and that for $\rho$ at a distance from a root of this coefficient not less than $\delta$ the coefficient is in absolute value greater than $\left|e^{\rho \omega_{\mu}\left(a_{2}-a\right)}\right| K_{4}$, in which $K_{4}$ is a constant dependent only on $\delta$. For convenience let this coefficient be denoted by $\Delta_{1}$. Then the inequality is

and $\Delta$ is

$$
\left|\Delta_{1}\right|>\left|e^{\rho 10_{\mu}\left(a_{2}-a\right)}\right| K_{4}
$$

$$
\Delta=\left[\theta_{1}\right]+\left[\theta_{k}\right] e^{\rho w_{\mu}(b-a)}+\left(\rho w_{\mu}\right)^{\nu} \Delta_{1} .
$$

This can be written

$$
\Delta=\left(\rho w_{\mu}\right)^{\nu} \Delta_{1}\left(1+\frac{\left[\theta_{1}\right]+\left[\theta_{k}\right] e^{\rho w_{\mu}(b-a)}}{\left(\rho w_{\mu}\right)^{\nu} \Delta_{1}}\right) .
$$

Now in $R$ the exponentials are bounded. Hence, if in $R$ any region be drawn, such that no part of the boundary is at a distance from a root of $\Delta_{1}$ less than $\delta>0$, then on the boundary $\Delta_{1}$ remains uniformly away from zero, and if the region be far enough out the parenthesis in (18) has, on the boundary, the form $1+\eta$, where $|\eta|<1$. From this can be deduced the fact that the roots of $\Delta$ in $R$ are asymptotically equal to those of $\Delta_{1}$.

Outside of $R$ for that part of $S$ in which $R\left(\rho w_{\mu}\right)<0$ the roots of $\Delta$ are asymptotically equal to those of

$$
f(\rho) \equiv\left(\rho w_{\mu}\right)^{v}+\frac{\theta_{1}}{\theta_{2}} e^{-\rho w_{\mu}\left(a_{2}-a\right)}=0,
$$

and for that part of $S$ in which $R\left(\rho w_{\mu}\right)>0$ they are asymptotically equal to those of

$$
\left(\rho w_{\mu}\right)^{\nu}+\frac{\theta_{k}}{\theta_{k-1}} e^{\rho w_{\mu}\left(b-a_{k-1}\right)}=0 .
$$

To prove the first of these statements, write

$$
\frac{\Delta}{\Delta_{1}}=f(\rho)+\frac{\left[\theta_{1}\right]}{\Delta_{1}}-\frac{\theta_{1}}{\theta_{2}} e^{-\rho 10_{\mu}\left(a_{2}-a\right)}+\frac{\left[\theta_{k}\right] e^{\rho 10_{\mu}(b-a)}}{\Delta_{1}}
$$




$$
\begin{aligned}
& =f(\rho)+\frac{[0]-\theta_{1} \sum_{j=3}^{k-1}\left[\theta_{j}\right] e^{\rho w w_{\mu}\left(a_{j}-a_{2}\right)}}{\Delta_{1} \theta_{2}}+\frac{\left[\theta_{k}\right] e^{\rho 2 w_{\mu}(b-a)}}{\Delta_{1}} \\
& =f(\rho)\left(1+\frac{[0]}{\Delta_{1} \theta_{2} f(\rho)}-\frac{\theta_{1} \sum_{j=3}^{k-1}\left[\theta_{j}\right] e^{\rho v_{\mu}\left(a_{j}-a_{2}\right)}}{\Delta_{1} \theta_{2} f(\rho)}+\frac{\left[\theta_{k}\right] e^{\rho w w_{\mu}(b-a)}}{\Delta_{1} f(\rho)}\right) .
\end{aligned}
$$

The roots of $f(\rho)$ have already been found, for it is the $f(\rho)$ of (11). To prove the roots of $\Delta$ asymptotically equal to those of $f(\rho)$, it is obviously sufficient to prove that the parenthesis above has the form $1+\eta$, for $\rho$ on the boundary of any region outside of $R$ and in that part of $S$ where $R\left(\rho w_{\mu}\right)$ $<0$, and such that the distance of any point of the boundary from a root of $f(\rho)$ is not less than $\delta>0$, provided that this region is taken far enough out from the origin. For such values of $\rho$ the inequalities (12), (14), and (17) hold, and they will be used to show that the parenthesis has that form. From (14) and (17) is obtained $\left|f(\rho) \Delta_{1}\right|>K_{1} K_{4}$, and since the symbol [0] is the same thing as $E / \rho$ it is evident that the term [0]/ $\Delta_{1} \theta_{2} f(\rho)$ is small for $\rho$ large. In the next term the same inequality on $f(\rho) \Delta_{1}$ is used for $T$ and $V$, since in these regions, for $\rho$ large, $R\left(\rho w_{\mu}\right)$ is large and negative, and the numerator, and so the whole term, is small. For $U$ the inequality $|f(\rho)|$ $\geqq\left(1-e^{-\sigma}\right)\left|\left(\rho w_{\mu}\right)^{\nu}\right|$ from (12) is used for $f(\rho)$. The remainder of the term may be written in the form

$$
E e^{-\rho w_{\mu}\left\{\left(a_{2}-a\right)-\left(a_{5}-a_{2}\right)\right\}}=E e^{-\rho w_{\mu}\left(a_{2}-a\right)\left[1-\left(a_{\xi}-a_{2}\right) /\left(a_{2}-a\right)\right]} .
$$

Now in $U$ the inequality

$$
\left|\left(\rho w_{\mu}\right)^{\nu}\right| \geqq e^{\sigma}\left|\theta_{a} e^{-\rho w_{\mu}\left(a_{2}-a\right)}\right|
$$

holds, from which it follows that

$$
\left|e^{-\rho w_{\mu}\left(a_{2}-a\right)\left[1-\left(a_{8}-a_{2}\right) /\left(a_{2}-a\right)\right]}\right| \leqq\left|\left(\rho w_{\mu}\right)^{v\left[1-\left(a_{8}-a_{2}\right) /\left(a_{2}-a\right)\right]}\right| \bar{K},
$$

where $\bar{K}$ is an absolute constant. The exponent of this power of $\rho$ is certainly less than $\nu$, so that because of the factor $\left|\left(\rho w_{\mu}\right)^{\nu}\right|$ from $f(\rho)$ in the denominator, the term approaches zero as $\rho$ becomes infinite. By reason of inequality (17) the last term of the parenthesis can obviously be written $E / f^{\prime}(\rho)$. In $T$ and $V$ inequality (16) shows that this is small for large values of $\rho$, while the inequality $|f(\rho)| \geqq\left(1-e^{-\sigma}\right)\left|\left(\rho w_{\mu}\right)^{\nu}\right|$ from (12) serves the same purpose for $U$. Thus the parenthesis has been shown to be of the required form for the values of $\rho$ in question, and so the roots of $\Delta$ are asymptotically equal to those of $f(\rho)$ for that part of $S$ outside of $R$ for which $R\left(\rho w_{\mu}\right)<0$.

The proof for the remaining part of $S$ on the other side of $R$ is similar. 
It is to be noted that the above reasoning applies to the whole of that part of $S$ in which $R\left(\rho w_{\mu}\right) \leqq 0$, even including the strip $R$, provided that $\rho$ is at a distance greater than $\delta$ from a root of $f(\rho)$ or $\Delta_{1}$, and is large enough. Hence from (19) can be obtained the following inequality which holds for all $\rho$ 's in absolute value greater than a certain $\bar{\rho}$ and at a distance from a root of $\Delta$ greater than $\delta$ :

$$
|\Delta|>\left|\Delta_{1}\right| \cdot|f(\rho)| K_{5},
$$

in which $K_{5}$ is a constant depending only on $\delta$.

In particular this shows, by reason of inequalities (14) and (17), that if in this region $\rho$ remains uniformly away from a root, then $\Delta$ remains uniformly away from zero.

Another inequality for $\Delta$ can easily be deduced from the above. First replace $\left|\Delta_{1}\right|$ by $\left|e^{\rho \omega_{\mu}\left(a_{2}-a\right)}\right| K_{4}$ from inequality (17). Then for the region $U$ replace $|f(\rho)|$ by $\left(1-e^{-\sigma}\right)\left|\left(\rho w_{\mu}\right)\right|^{\nu}$ from (12), and for the regions $T$ and $V$ replace it by $\left|e^{-\rho w_{\mu}\left(a_{2}-a\right)}\right| K_{1}$ from (14). But in the regions $T$ and $V$ the exponential can be replaced in this inequality by a constant times $\left|\left(\rho w_{\mu}\right)\right|^{\nu}$, so that finally the following is obtained:

$$
|\Delta|>\left|\left(\rho w_{\mu}\right)^{\nu} e^{\rho v_{\mu}\left(a_{2}-a\right)}\right| K_{6},
$$

in which $K_{6}$ is a constant depending only on $\delta$, and the inequality holds for all values of $\rho$ greater in absolute value than a certain $\bar{\rho}$ and at a distance from a root of $\Delta$ greater than $\delta$.

Even order case. When $n$ is even the substitution of the asymptotic expressions for the $W$ 's in the determinant $D(\lambda)$ gives

$$
D(\lambda) \equiv \prod_{i=1}^{n} \rho^{h_{i}} \prod_{j=\mu+2}^{n} e^{\rho x_{j}(b-a)} \Delta,
$$

in which $\Delta$ is that determinant which has for the element in the $i$ th row and the $j$ th column, $\left[\alpha_{1 i}\right] w_{j}^{h_{i}}$ for $j=1,2, \cdots, \mu-1$, and $\left[\alpha_{k i}\right] w_{j}^{h_{i}}$ for $j=\mu+2$, $\mu+3, \cdots, n$, and

$$
\begin{array}{r}
D_{i} \equiv w_{\mu}^{h_{i}}\left(\left[\alpha_{1 i}\right]+\left[\alpha_{k i}\right] e^{\rho v_{\mu}(b-a)}+\sum_{l=2}^{k-1}\left(\rho w_{\mu}\right)^{h_{i}-h_{i}}\left[\alpha_{l i}\right] e^{\rho o_{\mu}(a l-a)}\right) \quad(j=\mu), \\
H_{i} \equiv\left(-w_{\mu}\right)^{h_{i}}\left(\left[\alpha_{1 i}\right]+\left[\alpha_{k i}\right] e^{-\rho o_{\mu}(b-a)}+\sum_{l=2}^{k-1}\left(-\rho w_{\mu}\right)^{h_{i l}-h_{i}}\left[\alpha_{l i}\right] e^{-\rho o_{\mu}(a l-a)}\right) \\
(j=\mu+1)
\end{array}
$$

where $n=2 \mu$.

It is evident from the definition of the bracket symbol that in general this determinant expression for $\Delta$ can be expanded in the form

$$
\Delta \equiv P_{1}\left(\rho w_{\mu}\right)^{2 \nu}+P_{2}\left(\rho w_{\mu}\right)^{\nu}+P_{3},
$$

where the highest and lowest order terms of the $P$ 's are 


$$
\begin{aligned}
& P_{1}:\left[\theta_{11}\right] e^{\rho \omega_{\mu}\left(a_{k-1}-a_{2}\right)} \quad \text { and } \quad\left[\theta_{12}\right] e^{-\rho{ }^{2} \mu^{(}\left(a_{k-1}-a_{2}\right)} \text {; } \\
& P_{2}:\left[\theta_{21}\right] e^{\rho \omega_{\mu}\left(b-a_{2}\right)} \text { and }\left[\theta_{22}\right] e^{-\rho w_{\mu}\left(b-a_{2}\right)}, \quad \text { if } \quad b-a_{2}>a_{k-1}-a \text {, } \\
& \text { or }\left[\theta_{31}\right] e^{\rho w_{\mu}\left(a_{k-1}-a\right)} \text { and }\left[\theta_{32}\right] e^{-\rho 10_{\mu}\left(a_{k-1}-a\right)}, \quad \text { if } \quad b-a_{2}<a_{k-1}-a \text {; } \\
& P_{3}:\left[\theta_{41}\right] e^{\rho w_{\mu}(b-a)} \text { and }\left[\theta_{42}\right] e^{-\rho w_{\mu}(b-a)} \text {. }
\end{aligned}
$$

The number $\nu$ is the exponent of the highest power of $\rho$ appearing in a $D_{i}$ or $H_{i}$.

We shall now prove the

Theorem. If none of the $\theta^{\prime} s$ in (23) are zero, then $D(\lambda)$ has an infinite number of roots, and there exist two absolute constants $\omega$ and $\Omega$ such that the number of roots of $D(\lambda)$ in any circle about the origin of radius $r$ is less than $\Omega r$ and greater than $\omega r$, and finally for values of $\lambda$ uniformly away from its roots, $D(\lambda)$ remains uniformly away from zero.

This last statement is given more explicitly by inequality (25).

Should the number $\nu$ be negative, $\Delta$ reduces formally to Birkhoff's even order case, ${ }^{*}$ and the problem of distribution of roots will not be considered farther here. If $\nu$ is zero, $\Delta$ becomes merely a sum of exponentials which when multiplied through by the non-vanishing factor $e^{p \mu_{\mu}(b-a)}$ all have real parts of the same sign, and so are reduced to the form (8) already treated. In either of these cases the formal expansion (22) is not needed. But when $\nu$ is positive we make use of this expansion, which tacitly assumes that at least two of the $D_{i}$ 's and $H_{i}$ 's actually involve this power of $\rho$. This is, of course, equivalent to assuming that the highest order derivative in the auxiliary conditions at points other than the end points appears in at least two conditions. We also assume that none of the $\theta$ 's in (23) are zero, which is to say that certain of the determinants from the matrix of coefficients of the auxiliary conditions must not vanish. The method here used can be applied equally well with very slight modifications to many of the cases thus excluded; but a detailed study of the more specialized cases is beyond the scope of this paper.

The more general even order case, then, is treated as follows: The function $P_{1}$ can be reduced to the type (8) by multiplying through by $e^{\rho w_{\mu}\left(a_{k-1}-a_{2}\right)}$ and making the proper substitution. Its roots, then, all lie in a region $R$, and the proof that the roots of $\Delta$ in $R$ are asymptotically equal to those of $P_{1}$ is immediate. To find the roots outside of $R$ write

$$
\frac{\Delta}{P_{1}}=\left(\rho w_{\mu}\right)^{2 \nu}+\left(\rho w_{\mu}\right)^{\nu} \frac{P_{2}}{P_{1}}+\frac{P_{3}}{P_{1}}
$$

The line $R\left(\rho w_{\mu}\right)=0$ is now not the bisecting ray of each of the $S$ regions

\footnotetext{
* Birkhoff, loc. cit., p. 387.
} 
as in the odd order case, but the line of demarcation between two of them. Hence the region $R$ overlaps the t wo $S$ regions into which the $\lambda$-plane is carried by the transformation $\lambda=\rho^{n}$. Consider first the region $S_{1}$ in which $R\left(\rho w_{\mu}\right)$ $<0$. The above expression can be written in the form

$$
\begin{aligned}
\frac{\Delta}{P_{1}}=\left\{\left(\rho w_{\mu}\right)^{\nu}+\frac{\left[\theta_{32}\right]}{\left[\theta_{12}\right]} e^{-\rho w_{\mu}\left(a_{2}-a\right)}\right\}\left\{\left(\rho w_{\mu}\right)^{\nu}+\frac{\left[\theta_{42}\right]}{\left[\theta_{32}\right]} e^{-\rho w_{\mu}\left(b-a_{k-1}\right)}\right\} \\
+\left(\rho w_{\mu}\right)^{\nu} Q_{1}+Q_{2} .
\end{aligned}
$$

Suppose that $a_{2}-a$ is not equal to $b-a_{k-1}$ and, for convenience, let $a_{2}-a$ $>b-a_{k-1}$. Then the term of highest order in $Q_{1}$ is

and in $Q_{2}$,

$$
[\theta] e^{-\rho w_{\mu}\left(b-a_{k-1}\right)} \text {, }
$$

$$
[\theta] e^{-\rho v \theta_{\mu}\left(a_{2}-a\right)} \text {. }
$$

The expressions $Q_{1}$ and $Q_{2}$ are merely those terms which must be added to the product of the two factors in order to make the result equal to $\Delta / P_{1}$.

The roots of the two parentheses are found by the methods already developed, and it can be shown by means of inequalities of the type (12) and (14) that the roots of $\Delta$ in $S_{1}$ and outside of $R$ are asymptotically equal to those of the two parentheses.

Similarly the roots of $\Delta$ in $S_{2}$ and outside of $R$ are asymptotically equal to those of two parentheses of the same type, but with positive instead of negative exponents.

The following inequality is easily found for large values of $\rho$ which are in $S_{1}$ and at a distance from a root of $\Delta$ greater than $\delta$ :

$$
|\Delta|>\left|\left(\rho w_{\mu}\right)^{\nu}+\frac{\left[\theta_{32}\right]}{\left[\theta_{21}\right]} e^{-\rho w_{\mu}\left(a_{2}-a\right)}\right| \cdot\left|\left(\rho w_{\mu}\right)^{\nu}+\frac{\left[\theta_{42}\right]}{\left[\theta_{32}\right]} e^{-\rho w_{\mu}\left(b-a_{k-1}\right)}\right| \cdot\left|P_{1}\right| K_{7},
$$

in which $K_{7}$ is a constant depending only on $\delta$. If

the inequality for $\bar{\Delta}$ is

$$
\bar{\Delta}=\Delta e^{\rho \sigma_{\mu}(b-a)} \text {, }
$$

$$
|\bar{\Delta}|>\left|P_{1} e^{\rho w_{\mu}\left(a_{k-1}-a_{2}\right)}\right| \cdot \mid\left(\rho w_{\mu}\right)^{\nu} e^{\rho w_{\mu}\left(a_{2}-a\right)}
$$

$$
+\frac{\left[\theta_{32}\right]}{\left[\theta_{12}\right]}|\cdot|\left(\rho w_{\mu}\right)^{\nu} e^{\rho w_{\mu}\left(b-a_{k-1}\right)}+\frac{\left[\theta_{42}\right]}{\left[\theta_{32}\right]} \mid K_{7} .
$$

It can also be proved that for large values of $\rho$ in $S_{1}$ which are at a distance greater than $\delta$ from a root of $\bar{\Delta}$, the absolute value of $\bar{\Delta}$ remains greater than a positive constant depending only on $\delta$.

When $a_{2}-a=b-a_{k-1}$ the two systems of roots lie along two curves of the type (11) in which both have the same exponential, although in general 
the coefficients are different. If these coefficients should have the same absolute value the roots all lie along one curve, and if they are identical the roots are all double. An inequality like (24) holds in this case also, the only difference being in the $\theta$ 's and the value of $K_{7}$. From (24) it is easy to deduce the inequalities

$$
\begin{aligned}
|\bar{\Delta}| & >\left|\left(\rho w_{\mu}\right)^{2 \nu} e^{\rho v w_{\mu}\left(a_{2}-a\right)} e^{\rho 2 o_{\mu}\left(b-a_{k-1}\right)}\right| K_{8}, \\
& >\left|\left(\rho w_{\mu}\right)^{\nu} e^{\rho w_{\mu}\left(a_{2}-a\right)}\right| K_{9}, \\
& >\left|\left(\rho w_{\mu}\right)^{\nu} e^{\rho v w^{\prime}\left(b-a_{k-1}\right)}\right| K_{10},
\end{aligned}
$$

which hold under the same conditions as (24), and in which $K_{8}, K_{9}$, and $K_{10}$ depend only on $\delta$.

\section{The EXPANSION PROBLEM}

The expansion theorem is as follows:

TheOREM. Given $f(x)$, any real function which together with its first $\nu$ derivatives (or if $\nu<1$ with its first derivative) is continuous in the closed interval $(a, b)$, then its expansion in terms of the residues of the Green's function (3) converges to $f(x)$ at every interior point of $(a, b)$, if the auxiliary conditions have the following properties:

In the odd order case

Neither $\theta_{1}$ nor $\theta_{k}$ in (6) is zero.

If $\nu \geqq 1$ then in the coefficient of $\left(\rho w_{\mu}\right)^{\nu}$ neither $\theta_{2}$ nor $\theta_{k-1}$ is zero.

In the even order case

None of the $\theta$ 's in (23) are zero.

The intervals $b-a_{2}$ and $a_{k-1}-a$ are longer than any other of the subintervals of $(a, b)$ included between any two of the points $a, a_{2}, a_{3}, \cdots, a_{k-1}, b$.

The essential purpose of this last condition is merely that the second point from either end shall be farther away from that end than the first point from the other end is from that end. The conditions on the $\theta$ 's require merely that certain determinants from the matrix of the constants in the auxiliary conditions do not vanish. The condition on the $\theta$ 's in the even order case is stated in the above form in order to have it as simple as possible. If $\nu<1$ it is not necessary that all these $\theta$ 's be different from zero.

To prove the theorem it is necessary to consider separately the odd and even order cases. But before that is done it is convenient to introduce a new form of the expression for $\bar{G}(x, s ; \lambda)$ given in (2). If $\bar{G}$ be expanded in terms of the first row of the numerator determinant it can be written

in which

$$
\bar{G}(x, s ; \lambda) \equiv \pm \frac{1}{2} \sum_{i=1}^{n} y_{i}(x) \bar{y}_{i}(s),
$$

(26) $\quad \bar{y}_{i}(s) \equiv \frac{e^{-\rho v_{i}(s-a)} w_{i}}{n \rho^{n-1}}\left(1+\frac{\psi_{1}(s)}{\rho w_{i}}+\frac{\psi_{2}(s)}{\left(\rho w_{i}\right)^{2}}+\cdots+\frac{\psi_{n-1}(s)}{\left(\rho w_{i}\right)^{n-1}}+\frac{E}{\rho^{n}}\right)$. 
This expression for the $\bar{y}$ 's in so far as it differs from the one given by Birkhoff* is due to Milne. $\dagger$ In the work to follow, if $\nu<1$, Birkhoff's form would be sufficient, and the convergence proof is no more complicated than in the two point case. The complications necessary in case $\nu \geqq 1$, are due to the necessity of using the more explicit formulas of Milne.

The above form of $\bar{G}$ gives for $W_{i}(G \overline{)}$

$$
\begin{aligned}
W_{i}(\bar{G}) \equiv-\frac{1}{2} \sum_{j=1}^{n} W_{1 i}\left(y_{j}\right) \bar{y}_{j}(s) & \pm \frac{1}{2} \sum_{j=1}^{n} W_{2 i}\left(y_{j}\right) \bar{y}_{j}(s) \pm \cdots \\
\pm & \pm \frac{1}{2} \sum_{j=1}^{n} W_{k-1 i}\left(y_{j}\right) \bar{y}_{j}(s)+\frac{1}{2} \sum_{j=1}^{n} W_{k i}\left(y_{j}\right) \bar{y}_{j}(s),
\end{aligned}
$$

in which the plus sign is taken for values of $s$ to the left of the point at which the term in question applies, and the negative sign for values of $s$ to the right.

Odd order case. $R\left(\rho w_{\mu}\right) \leqq 0$. The convergence of the integral (5) is now to be studied. It has already been separated into the sum of two integrals, one in $S_{1}$ and one in $S_{2}$. In the odd order case it is necessary to separate each still farther into the sum of two integrals, one in that part of $S$ where $R\left(\rho w_{\mu}\right)<0$, and the other in that part where $R\left(\rho w_{\mu}\right)>0$. The successive paths of integration are arcs of circles $|\rho|=$ constant extending across both the regions $S_{1}$ and $S_{2}$, and so chosen that no point of such an arc is at a distance less than a preassigned $\delta>0$ from a root of $D$. That such arcs can be drawn in such a way that the sum of the orders of the roots between two successive arcs is not greater than $2 k$ when $\delta$ is chosen small enough, follows from the distribution of the roots of $\Delta$ as determined earlier in this paper. The parts of these arcs in $S_{1}$ and $S_{2}$ will be denoted by $\gamma_{1}$ and $\gamma_{2}$ as in (5). Let $S$ represent either of these regions. Then let $B$ be the point where the line $R\left(\rho w_{\mu}\right)=0$ in $S$ cuts one of these arcs, and let $A$ be the other end of the arc that crosses the region where $R\left(\rho w_{\mu}\right)<0$, and $C$ the other end of the arc that crosses the region where $R\left(\rho w_{\mu}\right)>0$. The convergence of the integral over the arc $A B$ will be studied first; that is, the integral

$$
I_{A B} \equiv \frac{1}{2 \pi i} \int_{A B} \int_{a}^{b} n \rho^{n-1} G\left(x, s ; \rho^{n}\right) f(s) d s d \rho .
$$

Before making use of the expression (3) for $G\left(x, s ; \rho^{n}\right)$ it is convenient to alter the last column by adding to it each of the columns 1 to $\mu$ multiplied by the term $\frac{1}{2} \bar{y}_{i}(s)$ with corresponding index, and each of the columns $\mu+1$ to $n$ multiplied by the term $-\frac{1}{2} \bar{y}_{i}(s)$ with corresponding index. The resulting last column is:

1st element, $\sum_{i=1}^{\mu} y_{i}(x) \bar{y}_{i}(s)$ for $y<x$, or $-\sum_{i=\mu+1}^{n} y_{i}(x) \bar{y}_{i}(s)$ for $y>x$;

* Birkhoff, loc. cit., p. 391.

$\dagger$ Milne, loc. cit., p. 167. 


$$
\begin{aligned}
& \text { (1+i)th element, } \\
& -\sum_{j=\mu+1}^{n} W_{1 i}\left(y_{j}\right) \bar{y}_{j}(s)+\left\{\begin{array}{c}
-\sum_{j=\mu+1}^{n} W_{2 i}\left(y_{j}\right) \bar{y}_{j}(s) \\
\sum_{j=1}^{\mu} W_{2 i}\left(y_{j}\right) \bar{y}_{j}(s)
\end{array}\right\}+\cdots \\
& +\left\{\begin{array}{c}
-\sum_{j=\mu+1}^{n} W_{k-1, i}\left(y_{j}\right) \bar{y}_{j}(s) \\
\sum_{j=1}^{\mu} W_{k-1, i}\left(y_{j}\right) \bar{y}_{j}(s)
\end{array}\right\}+\sum_{j=1}^{\mu} W_{k i}\left(y_{j}\right) \bar{y}_{j}(s),
\end{aligned}
$$

in which the upper term of each brace is taken for values of $s$ to the right of the point to which that term applies, and the lower for values of $s$ to the left.

The asymptotic solutions are now substituted in the expression $G$, and then the factor $\rho^{h_{i}}$ of $D$ is divided into the $(1+i)$ th row of the numerator, and the factor $e^{\rho w_{j}(b-a)}$ into the $j$ th column. The factor -1 is divided into the last column. Thus is obtained

$$
G\left(x, s ; \rho^{n}\right)=\frac{\bar{N}}{n \rho^{n-1}} \bar{\Delta},
$$

in which $\bar{N}$ is the determinant $\Delta$ bounded on the top by $e^{\rho w_{i}(x-a)}[1]$ with corresponding index for the columns 1 to $\mu$, and by $e^{\rho w_{i}(x-b)}$ [1] with corresponding index for the columns $\mu+1$ to $n$. On the right the $i$ th row is bounded by $F_{i}$ and the upper right-hand corner is filled in with $F$, where $F_{i}$ is

$$
-\sum_{j=\mu+1}^{n}\left[\alpha_{1 i} w_{j}^{h_{i}+1}\right] e^{\rho r_{j}(a-s)}+\sum_{l=2}^{k-1} \rho^{h_{l i}-h_{i}}\left\{\begin{array}{r}
- \\
\sum_{j=\mu+1}^{n}\left[\alpha_{l i} w_{j}^{h_{l i}+1}\right] e^{\rho w_{j}\left(a_{l}-s\right)} \\
\left.+\sum_{j=1}^{\mu} \alpha_{l i} w_{j}^{h_{l i}+1}\right] e^{\rho w_{j}\left(a_{l}-s\right)}
\end{array}\right\}
$$

and $F$ is

$$
\sum_{j=1}^{\mu} e^{\rho w_{j}(x-s)}\left[w_{j}\right], \text { for } s<x \text {, and }-\sum_{j=\mu+1}^{n} e^{\rho w_{j}(x-s)}\left[w_{j}\right] \text {, for } s>x ;
$$

and for convenience we give $B_{i}$, the $i$ th element in the $\mu$ th column of $\Delta: B_{i}$ is

$$
w_{\mu}^{h_{i}}\left(\left[\alpha_{1 i}\right]+\sum_{l=2}^{k-1}\left(\rho w_{\mu}\right)^{h_{l i}-h_{i}}\left[\alpha_{l i}\right] e^{\rho c_{\mu}\left(a_{l}-a\right)}+\left[\alpha_{k i}\right] e^{\rho w_{\mu}(b-a)}\right) .
$$

This expression for $G\left(x, s ; \rho^{n}\right)$ can now be substituted into (27), and since $s$ occurs only in the last column of $\bar{N}$, the function $f(s)$ can be multiplied into this column, and the $s$ integration performed. It is then to be noted that all terms of the determinant save possibly those in the $\mu$ th and last 
columns are bounded. Moreover the cofactor of $F$ is $-\Delta$, and so the determinant can be expanded to give

$$
\begin{aligned}
I_{A B} \equiv & \frac{1}{2 \pi i} \int_{A B} \int_{a}^{b}-F f(s) d s \\
& +\frac{1}{2 \pi i} \int_{A B}\left(\sum_{j=1}^{\mu=1} \sum_{r=1}^{n} \sum_{l=1}^{n} E_{j r l} e^{\rho w_{j}(x-a)} \frac{B_{r}}{\Delta} \int_{a}^{b} F_{l} f(s) d s\right) d \rho \\
& +\frac{1}{2 \pi i} \int_{A B}\left(\sum_{j=\mu+1}^{n} \sum_{r=1}^{n} \sum_{l=1}^{n} E_{j r l} e^{\rho w_{j}(x-b)} \frac{B_{r}}{\Delta} \int_{a}^{b} F_{l} f(s) d s\right) d \rho \\
& +\frac{1}{2 \pi i} \int_{A B}\left(\sum_{r=1}^{n} \frac{E_{r}}{\Delta} e^{\rho \rho \nu_{\mu}(x-a)} \int_{a}^{b} F_{r} f(s) d s\right) d \rho .
\end{aligned}
$$

It will be remembered that $E$ is a generic notation for functions of $\rho$ (and other variables) uniformly bounded for large values of $\rho$. In particular, in $(b)$ and $(c), E_{j r l}$ is identically zero for $r=1$.

The integral $(a)$ will be treated first. It is necessary to separate it into the sum of two integrals, one from $a$ to $x$ and the other from $x$ to $b$. The result of integrating these by parts with respect to $s$ gives $(\mu / 4 n) f(x)+\epsilon$ and $((\mu-1) / 4 n) f(x)+\epsilon$ respectively, for, as is easily seen,

$$
\int_{A B} \rho^{a} e^{\rho w_{j}(x-a)} d \rho, \quad \text { for } \quad j<\mu,
$$

and

$$
\int_{A B} \rho^{a} e^{\rho v_{j}(x-b)} d \rho, \quad \text { for } \quad j>\mu,
$$

approach zero as the radius of the arc $A B$ becomes infinite, for $\alpha$ any real constant, and $x$ distinct from $a$ and $b$. One also easily shows that

$$
\int_{A B} \frac{e^{\rho w_{\mu}(x-a)}}{\rho} d \rho
$$

approaches zero as this radius becomes infinite.

To evaluate $(b)$ and $(c)$ it is only necessary to notice that $1 / \Delta$ is bounded, while both $D_{r}$ and the $s$ integral are certainly less than a constant times $\rho^{n}$. Hence $(b)$ and $(c)$ reduce merely to bounded functions multiplied into integrals of the type (31), and so approach zero as the radius of $A B$ becomes infinite.

The evaluation of $(d)$ is more complicated. We now make use of the more explicit asymptotic solutions of Milne, and his expressions for the $\bar{y}$ 's, (26). In the formula for $F_{i}$ in (29), the square bracket $\left[\alpha_{l i} w_{j}^{h_{b}+1}\right]$, for example, has the more explicit form

$$
\left[\alpha_{l i} w_{j}^{h_{l}+1}\right]=w_{j}^{h_{h}+1}\left(\alpha_{l i}+\sum_{r=1}^{n-1} \frac{\alpha_{l i r}(s)}{\left(\rho w_{j}\right)^{r}}+\frac{E}{\rho^{n}}\right) .
$$


Consider now

$$
\int_{a}^{b} F_{i} f(s) d s
$$

It can be broken up into the sum of several integrals, since $F_{i}$ is a sum. Of these integrals consider first the one with the exponential involving $a_{l}(l \neq 1$, $k)$. When the more explicit form of the bracket symbol given above is used this becomes

$$
\begin{aligned}
& \rho^{h_{l i}-h_{i}}\left(\int_{a}^{a_{l}} \sum_{j=1}^{\mu} \alpha_{l i} w_{j}^{h_{l l}+1} e^{\rho w_{j}\left(a_{l}-s\right)} f(s) d s\right. \\
& \quad+\int_{a_{l}}^{b}-\sum_{j=\mu+1}^{n} \alpha_{l i} w_{j}^{h_{l i}+1} e^{\rho w_{j}\left(a_{l}-s\right)} f(s) d s \\
& +\int_{a}^{a_{l}} \sum_{j=1}^{\mu} \sum_{r=1}^{n-1} \frac{\alpha_{l i r}(s)}{\left(\rho w_{j}\right)^{r}} e^{\rho w_{j}\left(a_{l}-s\right)} w_{j}^{h_{l}+1} f(s) d s \\
& \quad+\int_{a_{l}}^{b}-\sum_{j=\mu+1}^{n} \sum_{r=1}^{n-1} \frac{\alpha_{l i r}(s)}{\left(\rho w_{j}\right)^{r}} e^{\rho w_{j}\left(a_{l}-s\right)} w_{j}^{h_{l i}+1} f(s) d s \\
& +\int_{a}^{a_{l}} \sum_{j=1}^{\mu} \frac{E}{\rho^{n}} e^{\rho w_{j}\left(a_{l}-s\right)} w_{j}^{h_{l l}+1} f(s) d s \\
& \left.\quad+\int_{a_{l}}^{s}-\sum_{j=\mu+1}^{n} \frac{E}{\rho^{n}} e^{\rho w_{j}\left(a_{l}-s\right)} w_{j}^{h_{l}+1} f(s) d s\right) .
\end{aligned}
$$

Now integrate (a) by parts and add the results, obtaining

$$
\begin{aligned}
\frac{\alpha_{l i}}{\rho}\left(f\left(a_{l}\right) \sum_{j=1}^{n}\right. & \left(-w_{j}^{h_{l i}}\right)+\sum_{j=1}^{\mu} w_{j}^{h_{l i}} f(a) e^{\rho w_{j}\left(a_{l}-a\right)}+\sum_{j=\mu+1}^{n} w_{j}^{h_{l i}} f(b) e^{\rho w_{j}\left(a_{l}-b\right)} \\
& \left.+\int_{a}^{a_{l}} \sum_{j=1}^{\mu} w_{j}^{h_{i}} e^{\rho w_{j}\left(a_{l}-s\right)} f^{\prime}(s) d s+\int_{a_{l}}^{b}-\sum_{j=\mu+1}^{n} w_{j}^{h_{l i}} e^{\rho w_{j}\left(a_{l}-s\right)} f^{\prime}(s) d s\right) .
\end{aligned}
$$

Since $n>h_{l i}>0$, the coefficient of $f\left(a_{l}\right)$ is zero. The remaining integrals can again be integrated by parts, ${ }^{*}$ and again the terms involving $a_{l}$ will drop out. This is repeated $h_{l i}-h_{i}$ times, which will give the factor $\alpha_{l i} / \rho^{h_{l i}-h_{i}}$ outside, and this is just enough to cancel the factor in (33). Then absolute values are taken, $f^{\left(h_{i}-h_{i}-1\right)}(s)$ is replaced by the upper limit to its absolute value, and the exponentials are again integrated, giving $E / \rho$. The total result may be written

$$
\sum_{j=1}^{\mu} E_{i j} \rho^{h_{l l}-h_{i}-1} e^{\rho v_{j}\left(a_{l}-a\right)}+\sum_{j=\mu+1}^{n} E_{i j} \rho^{h_{l i}-h_{i}-1} e^{\rho w_{j}\left(a_{l}-b\right)}+\frac{E_{i}}{\rho} .
$$

Next consider the $r$ th term of $(b)$. The factor $1 / \rho^{r}$ can be removed from

${ }^{*}$ It is to be noted that the number of derivatives here required has been provided for in the hypothesis of the expansion theorem, and also that if $\nu<1$ all this work with Milne's formulas is unnecessary. 
under the sign of integration, and $\alpha_{l i r}(s) f(s)$ can be considered as one function. The integral can then be treated as was $(a)$, but it requires only $h_{l i}-h_{i}-r$ integrations by parts and one further integration. The result can then be written in the same form as (34), though in this case, to be sure, the $E$ 's are not only bounded but actually approach zero. The terms $(c)$ are obviously of the form $E / \rho$, so that the whole of (33) can be written in the form (34). Finally a single integration by parts reduces the terms involving the points $a$ and $b$ to the form $E / \rho$, so that the integral

can be written as

$$
\int_{a}^{b} F_{i} f(s) d s
$$

$$
\sum_{l=2}^{k-1}\left(\sum_{j=1}^{\mu} E_{i j l} \rho^{h_{l i}-h_{i}-1} e^{\rho w_{j}\left(a_{l}-a\right)}+\sum_{j=\mu+1}^{n} E_{i j l} \rho^{h_{l i}-h_{i}-1} e^{\rho w_{j}\left(a_{l}-b\right)}\right)+\frac{E_{i}}{\rho} .
$$

When this is substituted in $(30 d)$ the last term here gives a result of the form (32). The other terms for $j \neq \mu$ give results of the type (31) with the value $a_{l}$ for $x$, and since for these terms $a_{l}$ is distinct from $a$ and $b$, the result of the $\rho$ integration approaches zero as the radius of $A B$ becomes infinite. The only terms left from (35) then are

$$
\sum_{l=2}^{k-1} E_{l} \rho^{h_{l i}-h_{i}-1} e^{\rho w_{\mu}\left(a_{l}-a\right)}
$$

which are multiplied by $(E / \Delta) e^{\rho w_{\mu}(x-a)}$ for the $\rho$ integration. If it can be proved that

$$
\sum_{l=2}^{k-1} \frac{E_{l} \rho^{h_{l}-h_{i}-1} e^{\rho w_{\mu}\left(a_{l}-a\right)}}{\Delta}
$$

is of the form $E / \rho$, then this last term will give a result of the form (32), and ( $30 d)$ will have been shown to approach zero as the radius of $A B$ becomes infinite. Now inequalities (16) and (21) prove this for $\nu>0$, while for $\nu \leqq 0$ the power of $\rho$ is negative while the exponential is bounded and $\Delta$ remains uniformly away from zero, and the same result is obtained.

To sum up the results, for the radius of $A B$ large

$$
I_{A B}=\frac{1}{4} f(x)+\epsilon .
$$

The work for the case $R\left(\rho w_{\mu}\right)>0$ runs along parallel to this in every detail, and the result is obtained that for the radius of $B C$ large

$$
I_{B C}=\frac{1}{4} f(x)+\epsilon .
$$

This result holds for both $S_{1}$ and $S_{2}$, so that (1) converges to $f(x)$ in the odd order case.

Even order case. In the even order case the paths of integration are the 
same as in the odd order case, but now there is no need of dividing farther the $\operatorname{arcs} \gamma_{1}$ and $\gamma_{2}$, since throughout each region $S$ the sign of the real part of $\rho w_{\mu}$ is unaltered. The region $S_{1}$ in which $R\left(\rho w_{\mu}\right) \leqq 0$ will be considered first, that is the integral

$$
I_{\gamma_{1}}=\frac{1}{2 \pi i} \int_{\gamma_{1}} \int_{a}^{b} n \rho^{n-1} G\left(x, s ; \rho^{n}\right) f(s) d s d \rho .
$$

In making use of the expression (3) for $G\left(x, s ; \rho^{n}\right)$ we now alter the last column by adding to it each of the columns 1 to $\mu$ multiplied by $\frac{1}{2} \bar{y}_{i}(s)$ with corresponding index, and each of the columns $\mu+1$ to $n$ multiplied by $-\frac{1}{2} \bar{y}_{i}(s)$ with corresponding index. The asymptotic solutions are then substituted, and the factor $\rho^{h_{i}}$ of $D$ is divided into the $(1+i)$ th row, and the factor $e^{\rho w_{j}(b-a)}$ into the $j$ th column. The factor $e^{\rho w_{\mu+1}(b-a)}$ is also divided out of the $(\mu+1)$ th column and out of $D$, thus leaving in the denominator

$$
\bar{\Delta} \equiv e^{\rho w_{\mu}(b-a)} \Delta,
$$

since $w_{\mu+1}=-w_{\mu}$. Thus $G\left(x, s ; \rho^{n}\right)$ is found to be equal to $1 /\left(n \rho^{n-1} \bar{\Delta}\right)$ multiplied into a determinant of order $n+1$ in which the element in the first row and $j$ th column is $e^{\rho r_{j}(x-a)}$ [1] for $j \leqq \mu$ and $e^{\rho \omega_{j}(x-b)}$ [1] for $\mu+1$ $\leqq j \leqq n$. The last element is $F$, given in (29). In the $(1+i)$ th row and $j$ th column the element is $\left[\alpha_{1 i} w_{j}^{h_{i}}\right]$ for $j<\mu,\left[\alpha_{k i} w_{j}^{h_{i}}\right]$ for $\mu+1<j \leqq n$, while for $j=\mu, \mu+1$, and $n+1$ the elements are respectively $D_{i}, \bar{H}_{i}$, and $F_{i}$, where $D_{i}$ is as given in (21a), while $\bar{H}_{i}$ is the $H_{i}$ given there, multiplied by $e^{\rho v_{\mu}(b-a)}$, and $F$ and $F_{i}$ are as given in (29).

The variable $s$ appears only in the last column of this determinant, whence $f(s)$ can be multiplied into this last column and the $s$ integration performed. Since all terms, save possibly the $D$ 's, $\bar{H}$ 's, and $F$ 's, are bounded, the expansion of the determinant takes the form

$$
\begin{aligned}
& I_{\gamma_{1}} \equiv \frac{1}{2 \pi i} \int_{\gamma_{1}} \int_{a}^{b}-F f(s) d s d \rho \\
& +\frac{1}{2 \pi i} \int_{\gamma_{1}, i, j, r=1}^{n}\left|\begin{array}{ccc}
D_{i} & \bar{H}_{i} & \int F_{i} f(s) d s \\
D_{j} & \bar{H}_{j} & \int F_{j} f(s) d s \\
D_{r} & \bar{H}_{r} & \int F_{r} f(s) d s
\end{array}\right|\left(\sum_{l=1}^{\mu-1} E_{l} e^{\rho^{\rho w_{l}(x-a)}}\right. \\
& \left.+\sum_{l=\mu+1}^{n} E_{l} e^{\rho w_{l}(x-b)}\right) d \rho
\end{aligned}
$$

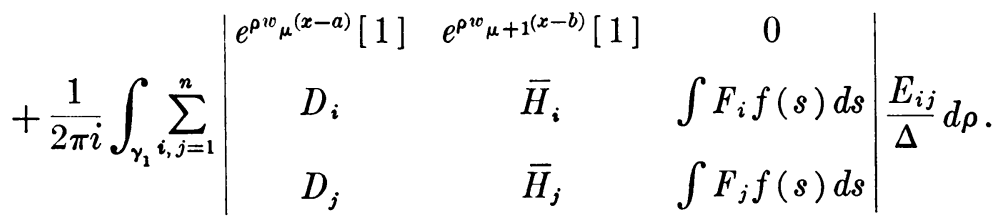


By comparing $(a)$ with the similar integral in the odd order case it is obvious that it approaches the limit $f(x) / 2$ as the radius of $\gamma_{1}$ becomes infinite. In $(b)$ the determinants can be written in the form $E \rho^{a}$, where $\alpha<3 n$. Since in the parenthesis the exponents have negative real parts on $\gamma_{1},(b)$ falls under the type (31), and so approaches zero as the radius of $\gamma_{1}$ becomes infinite. Finally $(c)$ is expanded in terms of the top row of the determinant, and the result is treated in a manner similar to that used in the odd order case. The expanded form is

$$
E_{1} e^{\rho v_{\mu}(x-a)} \sum_{i, j} \frac{1}{\bar{\Delta}}\left|\begin{array}{ll}
\bar{H}_{i} & \int F_{i} f d s \\
\bar{H}_{j} & \int F_{j} f d s
\end{array}\right|+E_{2} e^{\rho r_{\mu+1}(x-b)} \sum_{i, j} \frac{1}{\bar{\Delta}}\left|\begin{array}{ll}
D_{i} & \int F_{i} f d s \\
D_{j} & \int F_{j} f d s
\end{array}\right| .
$$

The formula for $\int F_{i} f(s) d s$ is given in (35), but a more explicit form for the $E$ terms in that expression is now needed. From (28) can be obtained directly a formula for $\int F_{i} f(s) d s$. It is

$$
\begin{aligned}
& \frac{\rho^{h_{i}}}{n \rho^{n-1}} \int_{a}^{b} F_{i} f(s) d s=-\sum_{j=\mu+1}^{n} W_{1 i}\left(y_{j}\right) \int_{a}^{b} \bar{y}_{j}(s) f(s) d s \\
& +\sum_{l=2}^{k-1}\left\{\begin{array}{c}
-\sum_{j=\mu+1}^{n} W_{l i}\left(y_{j}\right) \int_{a}^{b} \bar{y}_{j}(s) f(s) d s \\
\sum_{j=1}^{\mu} W_{l i}\left(y_{j}\right) \int_{a}^{b} \bar{y}_{j}(s) f(s) d s
\end{array}\right\}+\sum_{j=1}^{\mu} W_{k i}\left(y_{j}\right) \int_{a}^{b} \bar{y}_{j}(s) f(s) d s .
\end{aligned}
$$

By integrating by parts $\nu$ times,

$$
\int_{\sigma}^{\tau} e^{-\rho w_{j}(\delta-a)} f(s) d s=\frac{e^{-\rho w_{j}(\tau-a)}}{-\rho w_{j}}[f(\tau)]+\frac{e^{-\rho w_{j}(\sigma-a)}}{\rho w_{j}}[f(\sigma)]+\frac{E}{\rho^{\nu+1}},
$$

where

$$
[f(\tau)] \equiv f(\tau)+\frac{f^{\prime}(\tau)}{\rho w_{j}}+\cdots+\frac{f^{(\nu-1)}(\tau)}{\left(\rho w_{j}\right)^{\nu-1}},
$$

and $[f(\sigma)]$ has the same form. And now this can be applied to each term of $\bar{y}_{j}(s)$ in (26) with the result

$$
\frac{n \rho^{n-1}}{w_{j}} \int_{\sigma}^{\tau} \bar{y}_{j}(s) f(s) d s=\frac{e^{-\rho w_{j}(\tau-a)}}{-\rho w_{j}}[h(\tau)]+\frac{e^{-\rho w_{j}(\sigma-a)}}{\rho w_{j}}[h(\sigma)]+\frac{E}{\rho^{\nu+1}},
$$

where the symbol $[h(\tau)]$ has the more explicit form

$$
[h(\tau)]=h(\tau)+\frac{h_{1}(\tau)}{\rho w_{j}}+\frac{h_{2}(\tau)}{\left(\rho w_{j}\right)^{2}}+\cdots+\frac{h_{\nu-1}(\tau)}{\left(\rho w_{j}\right)^{\nu-1}},
$$

and $[h(\sigma)]$ is the same with the value $\sigma$ substituted in place of $\tau$. The function $h$ is the function $f$, but a different notation is introduced because 
the functions $h_{r}$ that enter are not precisely the derivatives of $f$. The essential thing in the expression is the fact that the functions $h_{r}$ are independent of $j$. When this result is used in (37) and the asymptotic solutions are substituted, the result must be of the form (35). Then by comparing the two forms the more explicit form for the $E$ terms in (35) is found:

$$
\begin{aligned}
\int F_{i} f(s) d s \equiv \sum_{l=2}^{k-1}\left(\sum_{j=1}^{\mu} w_{j}^{h_{l i}}\left[\alpha_{l i}\right][h(a)] \rho^{h_{l i}-h_{i}-1} e^{\rho w_{j}\left(a_{l}-a\right)}\right. \\
\left.\quad+\sum_{j=\mu+1}^{n} w_{j}^{h_{h i}}\left[\bar{\alpha}_{l i}\right][h(b)] \rho^{h_{l i}-h_{i}-1} e^{\rho w_{j}\left(a_{l}-a\right)}\right)+\frac{E}{\rho},
\end{aligned}
$$

in which $\left[\alpha_{l i}\right]$ is identical with the $\left[\alpha_{l i}\right]$ appearing in $D_{i}$ (i. e., when the bracket symbol is written out in full for any value of $m$, all corresponding terms as well as the $E$ terms are identical). In the same way $\left[\bar{\alpha}_{l i}\right]$ is identical with the $\left[\alpha_{l i}\right]$ appearing in $\bar{H}_{i}$. This evaluation of the integrals is now substituted in (36), and each determinant there can be written as the sum of several, according to the terms of (38). Since from (25) the expressions $D_{i} / \bar{\Delta}$ and $\left(H_{i} / \bar{\Delta}\right)$ are bounded on $\gamma_{1}$, the determinants which involve the last column $E / \rho$ are themselves of the form $E / \rho$, and that part of (36) is of the form (32), and so $^{\circ}$ converges to zero as the radius of $\gamma_{1}$ becomes infinite. On the other hand the terms involving exponentials other than $w_{\mu}$ or $w_{\mu+1}$ reduce to the form (31), with one of the constants $a_{l}(l \neq 1, k)$ in place of $x$. Hence these also approach zero. All that is left is then

where

$$
E_{1} e^{\rho w_{\mu}(x-a)} \sum_{i, j} \frac{1}{\bar{\Delta}}\left|\begin{array}{ll}
\bar{H}_{i} & N_{i} \\
\bar{H}_{j} & N_{j}
\end{array}\right|+E_{2} e^{\rho w_{\mu+1}(x-b)} \sum_{i, j} \frac{1}{\bar{\Delta}}\left|\begin{array}{ll}
D_{i} & N_{i} \\
D_{j} & N_{j}
\end{array}\right|,
$$

$$
\begin{aligned}
N_{i} \equiv \sum_{l=2}^{k-1} w_{\mu}^{h_{l i}}\left[\alpha_{l i}\right][h(a)] \rho^{h_{l i}-h_{i}-1} e^{\rho w_{\mu}\left(a_{l}-a\right)} & \\
& +\sum_{l=2}^{k-1} w_{\mu+1}^{h_{l_{i}}}\left[\bar{\alpha}_{l i}\right][h(b)] \rho^{h_{l i}-h_{i}-1} e^{\rho w_{\mu+1}\left(a_{l}-b\right)} .
\end{aligned}
$$

By inequality (25) the expression $\left(N_{i} / \bar{\Delta}\right)$ is of the form $E / \rho$. So when the values of $D_{i}$ and $\bar{H}_{i}$ are substituted from $(21 a)$ the first two terms of each give expressions that reduce to the form (32) and so approach zero. What still remains is

$$
\frac{E_{1} e^{\rho v_{\mu}(x-a)}}{\bar{\Delta}} \sum_{i, j}\left|\begin{array}{ll}
A_{b i} & B_{a i}+B_{b i} \\
A_{b j} & B_{a j}+B_{b j}
\end{array}\right|+\frac{E_{2} e^{\rho v 0_{\mu+1}(x-b)}}{\bar{\Delta}}\left|\begin{array}{ll}
A_{a i} & B_{a i}+B_{b i} \\
A_{a j} & B_{a j}+B_{b j}
\end{array}\right|
$$

where

$$
A_{a i} \equiv \sum_{l=2}^{k-1}\left[\alpha_{l i}\right] e^{\rho{ }^{\prime c_{\mu}}\left(a_{l}-a\right)}\left(\rho u_{\mu}\right)^{h_{l i}-h_{i}} w_{\mu}^{h_{i}}
$$




$$
\begin{aligned}
A_{b i} & \equiv \sum_{l=2}^{k-1}\left[\bar{\alpha}_{l i}\right] e^{\rho w_{\mu}\left(b-a_{l}\right)}\left(-\rho w_{\mu}\right)^{h_{l i} h_{i}}\left(-w_{\mu}\right)^{h_{i}}, \\
B_{a i} & \equiv \sum_{l=2}^{k-1}\left[\alpha_{l i}\right][h(a)] \rho^{h_{l i}-h_{i}-1} e^{\rho l c_{\mu}\left(a_{l}-a\right)} w_{\mu}^{h_{l i}}, \\
B_{b i} & \equiv \sum_{l=2}^{k-1}\left[\bar{\alpha}_{l i}\right][h(b)] \rho^{h_{l i}-h_{i}-1} e^{\rho l{ }^{l\left(b-a_{l}\right)}}\left(-w_{\mu}\right)^{h_{l i}} .
\end{aligned}
$$

These can be expanded into the sum of a large number of determinants. Such determinants as have the same exponential factor in both columns are zero, for example

$$
\left|\begin{array}{ll}
{\left[\bar{\alpha}_{l i}\right] e^{\rho w_{\mu}\left(b-a_{l}\right)}\left(-\rho w_{\mu}\right)^{h_{l i}-h_{i}}\left(-w_{\mu}\right)^{h_{i}}} & w_{\mu}^{h_{l i}}\left[\bar{\alpha}_{l i}\right][h(b)] \rho^{h_{l i}-h_{i}-1} e^{\rho w_{\mu}\left(b-a_{2}\right)} \\
{\left[\bar{\alpha}_{l j}\right] e^{\rho w_{\mu}\left(b-a_{l}\right)}\left(-\rho w_{\mu}\right)^{h_{l j}-h_{j}}\left(-w_{\mu}\right)^{h_{j}}} & w_{\mu}^{h_{l j}}\left[\bar{\alpha}_{l j}\right][h(b)] \rho^{h_{l j}-h_{j}-1} e^{\rho w_{\mu}\left(b-a_{2}\right)}
\end{array}\right|,
$$

for when the common factors

$$
e^{2 \rho w_{\mu}\left(b-a_{l}\right)}[h(b)]\left(-w_{\mu}\right)^{h_{l i}}\left(w_{\mu}\right)^{h_{l i}} \rho^{h_{l i}-h_{i}} \rho^{h_{l j}-h_{j}} \frac{1}{\rho}
$$

(it is to be noted that $[h(b)]$ is independent of the subscript) are removed, we have

$$
\left|\begin{array}{ll}
{\left[\bar{\alpha}_{l i}\right]} & {\left[\bar{\alpha}_{l i}\right]} \\
{\left[\bar{\alpha}_{l j}\right]} & {\left[\bar{\alpha}_{l j}\right]}
\end{array}\right|
$$

which is obviously zero, since the two terms in the same row of the determinant are identical.

Since $a_{2}-a$ and $b-a_{k-1}$ are the shortest of the intervals ending at $a$ or $b$, the terms that approach zero most slowly are $e^{\rho w_{\mu}\left(a_{2}-a\right)}$ and $e^{\rho w_{\mu}\left(b-a_{k-1}\right)}$, and each of the other determinants in (39) can be written

$$
E \rho^{2 \nu-1} e^{\rho w_{\mu}\left(a_{2}-a\right)} e^{\rho w_{\mu}\left(b-a_{k-1}\right)} .
$$

This divided by $\bar{\Delta}$ can, by inequality (25), be written $E / \rho$, whence (39) is of the form (32), and so approaches zero as the radius of $\gamma_{1}$ becomes infinite.

To sum up,

$$
I_{\gamma_{1}}=\frac{1}{2} f(x)+\epsilon
$$

for the radius of $\gamma_{1}$ sufficiently large.

The treatment of the integral in $S_{2}$, that is over $\gamma_{2}$ is, step for step, parallel to the above, and the result is obtained that for the radius of $\gamma_{2}$ sufficiently large

$$
I_{\gamma_{2}}=\frac{1}{2} f(x)+\epsilon .
$$

By combining the two results, the convergence of (1) is established in the even order case. 